\title{
A MIN-MAX APPROACH TO THE MULTIDIMENSIONAL NONUNIFORM FFT: APPLICATION TO TOMOGRAPHIC IMAGE RECONSTRUCTION
}

\author{
Jeffrey A. Fessler \\ EECS Department \\ The University of Michigan \\ fessler@umich.edu
}

\author{
Bradley P. Sutton \\ BME Department \\ The University of Michigan \\ bpsutton@umich.edu
}

\begin{abstract}
The FFT is used widely in signal processing for efficient computation of the Fourier transform (FT) over a set of uniformly spaced frequency locations. However, in many applications, one requires nonuniform sampling in the frequency domain, i.e., a nonuniform FT. Several papers have described fast approximations for the nonuniform FT based on interpolating an oversampled FFT. This paper presents a method for the nonuniform FT that is optimal in a minmax sense. The proposed method minimizes the worst-case approximation error over all signals of unit norm. Unlike many previous methods for the nonuniform FT, the proposed method easily generalizes to multidimensional signals. We are investigating this method as a fast algorithm for computing the Radon transform in 2D iterative tomographic image reconstruction.
\end{abstract}

\section{INTRODUCTION}

The fast Fourier transform (FFT) is used ubiquitously in signal processing applications where uniformly spaced samples in the frequency domain are needed. The FFT requires only $O(N \log N)$ operations, whereas direct evaluation of the discrete Fourier transform requires $O\left(N^{2}\right)$ operations. However, a variety of applications require nonuniform sampling in the frequency domain; examples include magnetic resonance image (MRI) reconstruction and computation of the Radon transform by means of the Fourier slice theorem. Such problems require a nonuniform Fourier transform, yet one would like to retain the $O(N \log N)$ computational advantages of fast algorithms like the FFT, rather than resorting to brute-force evaluation of the nonuniform FT.

Recently several papers in the scientific computing literature have described methods for approximating the ID nonuniform FT by interpolating an oversampled FFT, beginning with [1] and including [2-9]. Such methods are

\footnotetext{
*This work was supported in part by NIH grant CA-60711, NSF grant BES-9982349, the UM Center for Biomedical Engineering Research, and the Whitaker Foundation.
}

often called the nonuniform FFT, or NUFFT. Most of these algorithms have been presented only for 1D signals, and many involve somewhat arbitrary choices for interpolation functions. In contrast, this paper presents a min-max approach to the interpolation problem derived from first principles. We derive the (shift-variant) interpolator that minimizes the worst-case approximation error over all signals of unit norm. This method generalizes naturally to multidimensional signals such as the medical imaging problems in $M R I$ and tomography that motivated this work.

We have applied our new NUFFT method to iterative tomographic image reconstruction problems. For most iterative reconstruction methods, each iteration requires computation of one "forward projection" and one "backprojection," where the forward projection is roughly a discretized evaluation of the Radon transform, and the backprojector is the transpose (i.e., adjoint) of the forward projector. The projection and backprojection steps traditionally involve operations such as computing the lengths of intersections between each tomographic ray and each image pixel. These operations are the principal computational bottleneck in iterative reconstruction methods. A variety of methods for accelerating this process have been proposed, see e.g., [10]. One natural approach is to use the Fourier-slice theorem, which relates the 1D FT of each projection to samples of the 2D FT of the object on a polar grid ${ }^{1}$. This approach was proposed by Stearns et al. for 3D image reconstruction [11-13], but largely abandoned thereafter due to unacceptable image artifacts that resulted from significant errors in conventional interpolation/gridding methods for converting between polar and Cartesian coordinates in the frequency space. Our proposed min-max approach to the NUFFT provides sufficiently increased accuracy to largely eliminate those artifacts, so that we can compare Fourier slice based projections with conventional projection methods in terms of computational requirements. (NUFFT methods allow the user to tradeoff computation time and approximation error.) Delaney and Bresler [14] proposed a clever iterative algo-

\footnotetext{
${ }^{1}$ Shift-invariance detector blur is easily included in this framework
} 
rithm that also uses frequency domain principles; however, it is restricted to a particular form of weighting matrix that is suboptimal for low-count PET scans. The NUFFT-based projector will allow us to use weighting matrices of the form needed in PET [15] (at the expense of greater computation than required by the algorithm in [14]). Space constraints prohibit display of iterative tomographic image reconstruction results; this paper derives the new NUFFT method and illustrates its application as a fast method for computing the Radon transform based on the Fourier-slice theorem.

\section{THEORY: 1D CASE}

For simplicity, we first describe our min-max approach in the 1D case. The basic idea is to first compute an oversampled FFT of the given signal, and then optimally interpolate onto the desired nonuniform frequency locations using a small local neighborhood in the frequency domain around each desired value.

The problem is as follows. We are given equally spaced object samples ${ }^{2} x_{n}$, for $n=-N / 2,-N / 2+1, \ldots, N / 2-1$ with corresponding FT

$$
X(\omega)=\cdot \sum_{n=-N / 2}^{N / 2-1} x_{n} e^{-i n \omega}
$$

We wish to compute the FT at a collection of (nonuniformly spaced) frequency locations $\left\{\omega_{m}\right\}$ :

$$
y_{m} \triangleq X\left(\omega_{m}\right)=\sum_{n=-N / 2}^{N / 2-1} x_{n} e^{-i n \omega_{m}}
$$

for $m=1,2, \ldots, M$. Directly evaluating (1) would require $O(M N)$ operations, which is undesirable. Fast computation of (1) is called the NUFFT.

Our NUFFT algorithm is as follows:

1. $O(K \log K)$ Choose a convenient $K \geq N$ and compute the ordinary $K$-point FFT of $\left\{x_{n}\right\}$ :

$$
X_{k}=X\left(\frac{2 \pi}{K} k\right)=\sum_{n=-N / 2}^{N / 2-1} x_{n} e^{-i \frac{2 \pi}{K} k n},
$$

for $k=0, \ldots, K-1$.

2. $O(J M)$ Interpolate the $X_{k}$ 's to approximate each $y_{n}$ using the $J$ nearest neighbors to $\omega_{m}$. The approximation, $\hat{y}_{m}$, to $y_{m}$ has the following linear form:

$$
\hat{y}_{m}=\sum_{k=0}^{K-1} X_{k} v_{m k}^{\star}=\left\langle\boldsymbol{X}, v_{m}\right\rangle
$$

${ }^{2}$ In imaging problems, indexing from $-N / 2$ to $N / 2-1$ is more natural than indexing from 0 to $N-1$. where $v_{m k}$ denotes the collection of interpolation coefficients and "*" denotes complex conjugate.

To contain computational requirements, we constrain $\boldsymbol{v}_{m}$ to have at most $J$ nonzero elements corresponding to the $J$ nearest neighbors to $\omega_{m}$ in the set $\left\{\frac{2 \pi}{K} k\right\}$. For $J$ odd, define the integer $k_{m}$ as follows ${ }^{3}$ :

$$
k_{m} \triangleq\left(\arg \min _{k}\left|\omega_{m}-\frac{2 \pi}{K} k\right|\right)-\frac{J+1}{2}
$$

Let $u_{m j}, j=1, \ldots, J$ denote the $J$ possibly nonzero entries of $v_{m}$. Then the interpolation formula (3) becomes

$$
\hat{y}_{m}=\sum_{j=1}^{J} X_{k_{m}+j} u_{m j}^{\star} .
$$

To use this formula, one must precompute the $J M$ interpolation coefficients $u_{m j}$, and the $M$ indices $\left\{k_{m}\right\}$. (By precomputing, we mean that the $u_{m j}$ 's depend only on the desired sample locations $\left\{\omega_{m}\right\}$, which are usually related to the underlying system of interest, rather than on the signal values $\left\{x_{n}\right\}$.)

The problem then becomes choosing the $u_{m j}$ 's such that $\hat{y}_{m}$ is an accurate approximation to $y_{m}$, and such that the $u_{m j}$ 's are relatively easy to precompute. Dutt and Rokhlin used Gaussian kemels for their interpolation method [1]. Nguyen and Liu [7] considered an interpolation of the form (4) without an explicit criterion for their choice of the $u_{m j}$ 's.

We adopt a min-max criterion for choosing the interpolation coefficients $u_{m j}$. For each desired sample location $\omega_{m}$, we determine the vector $\boldsymbol{u}_{m}=\left(u_{m 1}, \ldots, u_{m J}\right)$ that minimizes the worst case approximation error between $y_{m}$ and $\hat{y}_{m}$ over all signals with unit norm. This is a shiftvariant interpolation method since each desired frequency location has its own set of $J$ interpolation coefficients. Thus the storage requirements are $O(J M)$.

Mathematically, our min-max criterion is as follows:

$$
\min _{\boldsymbol{u}_{m_{i}} \in \mathbb{C}^{J}} \max _{\boldsymbol{x} \in \mathbb{C}^{N}:\|\boldsymbol{x}\| \leq 1}\left|\hat{y}_{m}-y_{m}\right| .
$$

Remarkably, this min-max problem has an analytical solution that we derive as follows. From (4) and (1), we have the following expression for the error:

$$
\left|\hat{y}_{m}-y_{m}\right|=\left|\sum_{j=1}^{J} X_{k_{m}+j} u_{m j}^{\star}-X\left(\omega_{m}\right)\right| .
$$

Using (2) and (6), this error expression becomes

$$
\sum_{j=1}^{J} u_{n j}^{\star} \sum_{n=-N / 2}^{N / 2-1} x_{n} e^{-i \frac{2 \pi}{K}\left(k_{n n}+j\right) n}-\sum_{n=-N / 2}^{N / 2-1} x_{n} e^{-i n \omega_{m}}
$$

${ }^{3} \mathrm{~A}$ similar formula with slightly different offset is needed for the case where $J$ is even. 


$$
\begin{gathered}
=\sum_{n=-N / 2}^{N / 2-1} x_{n}\left(\sum_{j=1}^{J} u_{m j}^{\star} e^{-i \frac{2 \pi}{K}\left(k_{m}+j\right) n}-e^{-i n \omega_{m}}\right) \\
=\left\langle\boldsymbol{x}, \boldsymbol{g}_{\boldsymbol{m}}\right\rangle,
\end{gathered}
$$

where $g_{m}$ is defined by

$$
g_{n}^{(m)} \triangleq \sum_{j=1}^{J} u_{m j} e^{i \frac{2 \pi}{K}\left(k_{m}+j\right) n}-e^{i n \omega_{m}}
$$

for $n=-N / 2, \ldots, N / 2-1$. In matrix-vector form:

$$
\boldsymbol{g}_{m}=\boldsymbol{D}^{(m)} \boldsymbol{W} \boldsymbol{u}_{m}-\boldsymbol{b}_{m},
$$

where $D^{(m)}$ is a $N \times N$ diagonal matrix with entries

$$
D_{n n}^{(m)}=e^{i \frac{2 \pi}{K} k_{m} n},
$$

$\boldsymbol{W}$ is a $N \times J$ matrix with entries $w_{n j}=e^{i \frac{2 \pi}{K} j n}$, and $b_{m}$ is a length- $N$ vector with entries $b_{n}^{(m)}=e^{i n \omega_{m}}$.

With these definitions, the min-max problem (5) becomes

$$
\min _{\boldsymbol{u}_{m} \in \mathbb{C}^{J}} \max _{\boldsymbol{x} \in \mathbb{C}^{N}:\|\boldsymbol{x}\| \leq 1}\left|\left\langle\boldsymbol{x}, \boldsymbol{g}_{m}\right\rangle\right|
$$

Applying the Cauchy-Schwarz inequality, the worst case object $\boldsymbol{x}$ is when $\boldsymbol{x}=\boldsymbol{g}_{m}^{\star} /\left\|g_{m}\right\|$, i.e.,

$$
\max _{\boldsymbol{x}:\|\boldsymbol{x}\|=1}\left|\left\langle\boldsymbol{x}, \boldsymbol{g}_{m}\right\rangle\right|=\left.\left|\left\langle\boldsymbol{x}, \boldsymbol{g}_{m}\right\rangle\right|\right|_{\boldsymbol{x}=\boldsymbol{g}_{m}^{*} /\left\|\boldsymbol{g}_{m}\right\|}=\left\|\boldsymbol{g}_{m}\right\| .
$$

Inserting this case into the preceding min-max problem (11) and applying (9) reduces the problem to the following:

$$
\min _{\boldsymbol{u}_{m} \in \mathbb{C}^{J}}\left\|\boldsymbol{D}^{(m)} \boldsymbol{W} \boldsymbol{u}_{m}-\boldsymbol{b}_{m}\right\| .
$$

The minimizer of this simple least-squares problem is:

$$
\begin{aligned}
\boldsymbol{u}_{m} & =\left(\boldsymbol{W}^{\prime} \boldsymbol{D}^{(m) \prime} \boldsymbol{D}^{(m)} \boldsymbol{W}\right)^{-1} \boldsymbol{W}^{\prime} \boldsymbol{D}^{(m) \prime} \boldsymbol{b}_{m} \\
& =\left(\boldsymbol{W}^{\prime} \boldsymbol{W}\right)^{-1} \boldsymbol{W}^{\prime} \boldsymbol{D}^{(m) \prime} \boldsymbol{b}_{m},
\end{aligned}
$$

using (10). Fortuitously, the inverse of the $J \times J$ matrix $\boldsymbol{W}^{\prime} \boldsymbol{W}$ is independent of frequency sample location so it is easily precomputed. Nguyen and Liu called this type of matrix a $(K / N, N, J)$ regular Fourier matrix [7]. Similar to [7], the entries of $W^{\prime} W$ here are given by

$$
\left[\boldsymbol{W}^{\prime} \boldsymbol{W}\right]_{l, j}=\sum_{n=-N / 2}^{N / 2-1} w_{n l}^{\star} w_{n j}=\kappa(j-l)
$$

where $\kappa(\cdot)$ denotes the following Dirichlet-like kernel:

$$
\kappa(t) \triangleq \epsilon^{-i \pi t / K} \frac{\sin \left(\frac{2 \pi}{K} t N / 2\right)}{\sin \left(\frac{2 \pi}{K} t / 2\right)} .
$$

Remarkably, in this min-max framework the final matrixvector product in (12) also simplifies to a closed-form expression as follows

$$
\begin{aligned}
{\left[\boldsymbol{W}^{\prime} \boldsymbol{D}^{(m) \prime} \boldsymbol{b}_{m}\right]_{j} } & =\sum_{n=-N / 2}^{N / 2-1} e^{-i \frac{2 \pi}{K} j n} e^{-i \frac{2 \pi}{K} k_{m} n} e^{i n \omega_{m}} \\
& =\kappa\left(\omega_{m} / \frac{2 \pi}{K}-k_{m}-j\right)
\end{aligned}
$$

which is essentially a Dirichlet-like function of the residual differences between the desired frequency sample locations and the nearest oversampled FFT grid points.

In summary, we precompute the min-max interpolation coefficients in (12) via the analytical results (13) and (14). This precomputation requires only $O\left(J^{2} M\right)$ operations. A key property of (13) and (14) is that they reduce the summations over $n$, thereby making the precomputing practical. As noted near (2) and (3), performing the interpolation itself to compute the NUFFT requires $O(K \log K)+O(J M)$ operations. An accuracy-computation time tradeoff is available through the choices for the oversampling factor $K / N$ and the neighborhood size $J$. Typically we use $K \approx 3 N$ and $J<10$, so the overall computational requirements are roughly $O(N \log N)$, akin to the FFT but with a larger constant. (The larger constant is an unavoidable consequence of needing accurate nonuniform frequency samples!)

\section{THEORY: 2D CASE}

Space constraints prohibit a full exposition of the multidimensional case. The overall approach is a natural generalization of the min-max framework described above. We oversample the 2D FFT in both directions, and find the minmax interpolator for each desired frequency location using the nearest $J \times J$ sample locations. The storage requirements are $O\left(J^{2} M\right)$.

\section{RESULTS}

We applied the 2D NUFFT in conjunction with the Fourierslice theorem to calculate the tomographic forward projection (i.e., Radon transform) of the ubiquitous Shepp-Logan digital phantom with $N \times N=128 \times 128$ pixels. For comparison, we also computed the projection using discretized strip integrals which are based on the areas of intersection of a strip corresponding to a PET detector width with each pixel [16]. (This system model for PET realistically accounts for the finite detector width rather than using overly idealized line integrals.) The sinogram size was 160 radial bins by 192 angles per CTI EXACT PET scanners.

We compared the conventional strip-integral projections vs the NUFFT-based projections for several values of the 
neighborhood size $J$ and the FFT oversampling factor $K / N$. For reasonable values of $J$ and $K / N$ the projections are indistinguishable when printed in grayscale or in profiles, so are not shown. The figure below shows the tradeoff between computation time (using Matlab's tic command on a $1 \mathrm{GHz}$ Pentium III running Linux) and percent RMS difference between the conventional approach and the NUFFTbased approach. We also examined the $l_{1}$ and $l_{\infty}$ norm differences which showed identical trends.

Using $J=6$ and $K=2 N$, the NUFFT approach is 4 times faster than the conventional approach in this geometry, yet yields a normalized RMS error of less than $0.15 \%$. In this case, precomputing the $\boldsymbol{u}_{m}$ 's required less than 8 seconds. (This precomputation depends only on the scanner geometry, and not the object, so needs only to be done once for a given tomographic system so its computation time is largely irrelevant.) A reduction by a factor of 4 in computation time would be quite significant for the practical use of iterative image reconstruction methods.

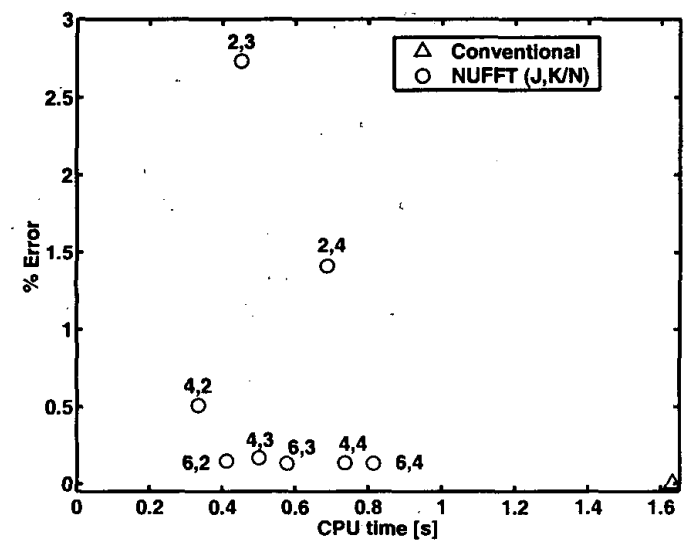

\section{DISCUSSION}

The proposed min-max method for the NUFFT should have applications in a variety of signal processing and imaging problems where nonuniform frequency samples are required.

We have used the usual norm in our min-max formulation; in some applications it may be useful to consider alternative norms. The general theory will easily accommodate any quadratic norm; however, whether simplifications of the form (13) and (14) appear may depend on the norm.

One remaining open problem is that the $J \times J$ matrix $\boldsymbol{W}^{\prime} \boldsymbol{W}$ becomes ill-conditioned as $J$ increases. We currently use a truncated SVD type of pseudo-inverse when such ill-conditioning appears (which is feasible since $J$ is typically 5-15). Perhaps a more sophisticated form of regularization of its inverse could improve accuracy.

Of course by duality, one could easily adapt the entire approach to problems where the frequency samples are uniform but the object samples are nonuniform.

\section{REFERENCES}

[1] A Dutt and V Rokhlin, "Fast Fourier transforms for nonequispaced data," SIAM J. Sci. Comp., vol. 14, no. 6, pp. 136893, Nov. 1993.

[2] C Anderson and M D Dahleh, "Rapid computation of the discrete Fourier transform," SIAM J. Sci. Comp., vól. 17, no. 4, pp. 913-9, 1996.

[3] G Beylkin, "On the fast Fourier transform of functions with singularities," Applied computational harmonic analysis, vol. 2, no. 4, pp. 363-81, Oct. 1995.

[4] A Dutt and V Rokhlin, "Fast Fourier transforms for nonequispaced data, II," Appl. and Comp. Harm. Anal., vol. 2, pp. 85-100, 1995

[5] Q H Liu and N Nguyen, "An accurate algorithm for nonuniform fast Fourier transforms (NUFFT's)," IEEE Microwave and Guided Wave Letters, vol. 8, no. 1, pp. 18-20, Jan. 1998.

[6] Qing Huo Liu and Xue Yuan Tang, "Iterative algorithm, for nonuniform inverse fast Fourier transform," Electronics Letters, vol. 34, no. 20, pp. 1913-4, Oct. 1998.

[7] Nhu Nguyen and Qing Huo Liu, "The regular Fourier matrices and nonuniform fast Fourier transforms," SIAM J. Sci. Comp., vol. 21, no. 1, pp. 283-93, 1999.

[8] G Steidl, "A note on the fast Fourier transforms for nonequispaced grids," Advances in computational mathematics, vol. 9, pp. 337-52, 1998.

[9] A F Ware, "Fast approximate Fourier transforms for irregularly spaced data," SIAM Review, vol. 40, no. 4, pp. 838-56, Dec. 1998.

[10] S Basu and Y Bresler, " $O\left(n^{2} \log _{2} n\right)$ filtered backprojection reconstruction algorithm for tomography," IEEE Tr. Im. Proc., vol. 9, no. 10, pp. 1760-73, Oct. 2000.

[11] C W Stearns, D A Chesler, and G L Brownell, "Threedimensional image reconstruction in the Fourier domain," IEEE Tr. Nuc. Sci., vol. 34, no. 1, pp. 374-8, Feb. 1987.

[12] C W Stearns, D A Chesler, and G L Brownell, "Accelerated image reconstruction for a cylindrical positron tomograph using Fourier domain methods," IEEE Tr. Nuc. Sci., vol. 37, no. 2, pp. 773-7, Apr. 1990.

[13] S Matej, "3D-FRP: direct Fourier reconstruction with Fourier reprojection for fully 3D PET," in Proc. IEEE Nuc. Sci. Symp. Med. Im. Conf., 2000, vol. ?

[14] A H Delaney and Y Bresler, "A fast and accurate Fourier algorithm for iterative parallel-beam tomography," IEEE $\mathrm{Tr}$. Im. Proc., vol. 5, no. 5, pp. 740-53, May 1996.

[15] J A Fessler, "Penalized weighted least-squares image reconstruction for positron emission tomography," IEEE Tr. Med. Im., vol. 13, no. 2, pp. 290-300, June 1994.

[16] J A Fessler, "ASPIRE 3.0 user's guide: A sparse iterative reconstruction library," Tech. Rep. 293, Comm. and Sign. Proc. Lab., Dept. of EECS, Univ. of Michigan, Ann Arbor, MI, 48109-2122, July 1995, Available from http: / / www. eecs.umich. edu/ fessler. 